\title{
Neurotypology of Sentence Comprehension: Cross-Linguistic Difference in Canonical Word Order Affects Brain Responses during Sentence Comprehension
}

\author{
Yosuke Hashimoto $^{1,2}$, Satoru Yokoyama ${ }^{*}, 1$ and Ryuta Kawashima ${ }^{1}$ \\ ${ }^{1}$ Institute of Development, Aging, and Cancer, Tohoku University, Japan \\ ${ }^{2}$ Akita International University, Okutsubakidai-1,9,3-2 Yuwatsubakigawa, Akita, Akita Prefecture 010-1211, Japan
}

\begin{abstract}
While a clear variability of canonical word order across languages has been found, such a finding is not reflected in recent neuroimaging studies of language processing. Languages having a canonical word order of SubjectObject-Verb (SOV) in a sentence make up approximately $43 \%$ of world languages, while languages having a SubjectVerb-Object (SVO) word order make up approximately 37\%. Sufficient attention has not been given to this typological difference in neuroimaging studies. In this article, we review neuroimaging studies of sentence processing to examine whether the typological difference of canonical word order in a sentence is represented in brain activation results or not. As a result of this literature survey, an effect from the difference in canonical word order was found to exist between SVO and SOV languages for brain activation during sentence comprehension. This effect was found mainly in the left inferior and middle frontal gyri, precentral gyrus, supplemental motor area, inferior and middle temporal gyri, temporal pole, hippocampus, and cerebellum. These results imply that a difference in canonical word order causes a different sentence processing pattern, as well as a different load in the working memory process.
\end{abstract}

Keywords: Typology, canonical word order, neuroimaging, sentence comprehension.

\section{INTRODUCTION}

So far, to examine how the human brain processes language, several medical/neuroimaging techniques have been used. Particularly, which brain area is associated with language processing has been examined. However, mainly due to limitations in terms of participant recruitment for such neuroimaging studies, cross-linguistic differences among languages have not been examined.

All over the world, approximately 7000 languages exist, each of which has different linguistic characteristics [1]. Language typology tries to uncover the universal characteristics among these numerous languages through several kinds of linguistic criteria. One important finding in this field is canonical word order [2]. Canonical word order at the clausal/sentential level is composed of three major constituents: $\mathrm{S}$ (subject), $\mathrm{O}$ (object), and $\mathrm{V}($ verb). For example, an English sentence usually consists of "I (S)", "like (V)", and "Sushi (O)" but "I (S) Sushi (O) like (V)" is grammatically incorrect. It is now widely known that at least $80 \%$ of the languages in the world are classified as either having Subject-Verb-Object (SVO) order or Subject-Object-Verb (SOV) order. Languages having SOV word order make up approximately $43 \%$ of languages in the world, while languages having SVO order make up approximately $37 \%$ [2]. Many researchers have come to this same conclusion about language and it is now claimed that the key contrast of

*Address correspondence to this author at the Institute of Development, Aging, and Cancer, Tohoku University, 4-1 Seiryo-machi, Aoba-ku, Sendaicity 980-8575, Japan; Fax: +81-22-717-7988; Tel: +81-22-717-7988; E-mails: yokoyama@idac.tohoku.ac.jp, satyok@gmail.com canonical word order is whether the language is head-initial (head is V here; SVO) or head-final (SOV) [3-6]. This difference in word order can predict various aspects of language processing. For example, while head-initial SVO languages have a rich verb-argument system, head-final SOV languages have a rich case-marking system $[3,4]$.

Such an important finding in linguistic typology has also affected psycholinguistic models of sentence comprehension. In particular, it has already been pointed out that a difference in canonical word order among languages affects sentence comprehension strategies $[5,6]$. In SVO languages, the head of the sentence or clause (i.e., predicate including the verb) appears at the early stage of the sentence, in particular, after the subject appears. Since, in SVO languages, the head can determine the entire structure of the sentence, the information of the head can be used at an early stage of sentence comprehension. Contrastively, in SOV languages, the head is placed at the end of the sentence; thus the information of the head cannot be used until the end of the sentence. In addition, in SOV languages, other arguments should be tentatively memorized until the head computes the entire sentence structure. Such a maintenance process requires a severe cognitive load on short-term (working) memory. In order to avoid this maintenance load, it is assumed that other arguments in the sentence are incrementally processed before the head appears. In contrast, since SVO languages can use the head information at an early stage, such an incremental process is not required because the memory load of the arguments is small. Consequently, due to the above difference between SVO and SOV word-order languages, the sentence comprehension strategies used between them should be different as well. As described above, the difference between 
the SVO and SOV languages interacts with the way essential grammatical relationships between noun phrases are marked. SVO languages tend to mark grammatical functions, such as subject and object, using word order (e.g., English and Chinese); in contrast, SOV languages tend to mark grammatical functions using case particle attachments (e.g., Japanese and Korean).

While a large number of neuroimaging studies of sentence comprehension have been performed, to our knowledge, they paid little attention to the typological difference in sentence comprehension. While several neuroimaging studies imply that a difference in canonical word order has some effect on sentence comprehension [6-8], these previous studies, which did not examine monolingual subjects but rather bilingual subjects and sign language users, provided only indirect evidence. The reason why there is no study directly examining the word order effect on sentence comprehension among languages is that it is difficult to directly compare brain activities during comprehension of different types of languages in terms of experimental design and collecting a sufficient number of native speakers of each language as participants. In this sense, literature review is one of the suitable ways to begin examining sentence comprehension. Since linguistic typological factors psycholinguistically affect sentence comprehension, as we pointed out above, it is reasonable to predict that differences among languages in canonical word order on sentence comprehension would be represented in functional brain imaging data.

In this paper, to investigate whether or not the difference in word order affects functional brain imaging data during sentence comprehension, we used a simple and explorative review in the following way: We firstly chose previous papers from PubMed, and the peak activation coordinates were listed. Then, we labeled and grouped the peaks by utilized language (SVO or SOV). Finally, the percentage of the activation for each anatomical region was summarized by categorization of the word order. The purpose of our paper was to survey previous neuroimaging findings to see the trend of the effect of the different word orders among languages used in the neuroimaging studies. Hence, in this paper, we did not perform any statistical tests using this data.

\section{METHODS}

Firstly, we chose literature which included a sentence comprehension condition in the participant's first language from papers indexed in Medline. We searched for literature in May of 2010 using "(fMRI OR PET) sentence (processing OR comprehension)" as the keywords. In order to choose the papers we used here, we excluded papers using sentence stimuli whose canonical word order we cannot clearly determine. Then, out of the papers appearing in the search results, we chose 12 papers which included 21 'sentence $v s$. word list' contrasts in total [10-20] (Table 1). This contrast can be adjusted to exclude the effect of a cross-linguistic difference in the word recognition process by subtracting the word list condition from the sentence comprehension condition in the brain imaging results. Hence, we assume that this contrast can effectively extract the language-based difference that word order has on brain activity. Out of the studies we collected, four were SVO language studies, and seven were SOV language studies. One of the SVO language studies included was English $(n=5)$ [10-14]. In the SOV language studies, Japanese $(n=7)[9,15-20]$ was included. We categorized the languages by 1) canonical word order (SVO or SOV), 2) experimental task/condition (e.g., sentence $v s$. word list), and 3 ) the peaks of the activated regions (e.g., the opercular part of the left inferior frontal gyrus). To label the activated regions in an objective manner, we used Masks for Region of Interest Analysis (MARINA) (http://www.bion. de/index.php?title=MARINA) [21] software, which was made using AAL-based labeling of anatomical regions of the brain. Using the list we created above, we summarized the percentage of the reported activation for each region to review the trend that typological difference in canonical word order had on brain activation patterns.

Table 1. Selected Papers for the Current Review

\begin{tabular}{|c|c|c|}
\hline \multirow{5}{*}{ SVO } & \multirow{5}{*}{ English } & Rogalsky et al. 2009 \\
\hline & & Humphries et al. 2006 \\
\hline & & Maguire et al. 2006 \\
\hline & & Vandenberghe et al. 2002 \\
\hline & & Bottini et al. 1994 \\
\hline \multirow{7}{*}{ SOV } & \multirow{7}{*}{ Japanese } & Yokoyama et al. 2006 \\
\hline & & Ikuta et al. 2006 \\
\hline & & Homae et al. 2002 \\
\hline & & Yokoyama et al. 2009 \\
\hline & & Kim et al. 2009 \\
\hline & & Homae et al. 2003 \\
\hline & & Hashimoto et al. 2002 \\
\hline
\end{tabular}

\section{RESULTS}

Our literature review results showed a different trend between SVO and SOV languages (Fig. 1 and Table 2) in the 'sentence $v s$. word list' contrast. The greatest difference between the SVO and SOV languages was found in the activation of the left inferior frontal gyrus. In our results, $86 \%$ of neuroimaging studies using SOV languages reported left inferior frontal activation, while $57 \%$ of neuroimaging studies using SVO languages reported left inferior frontal activation. Also, the SOV languages activated several left frontal regions (e.g., precentral gyrus) and the cerebellum more than the SVO languages did. Contrastively, the left inferior temporal gyrus and temporal pole have been reported to be more active in neuroimaging studies using SVO languages than in those using SOV languages. 

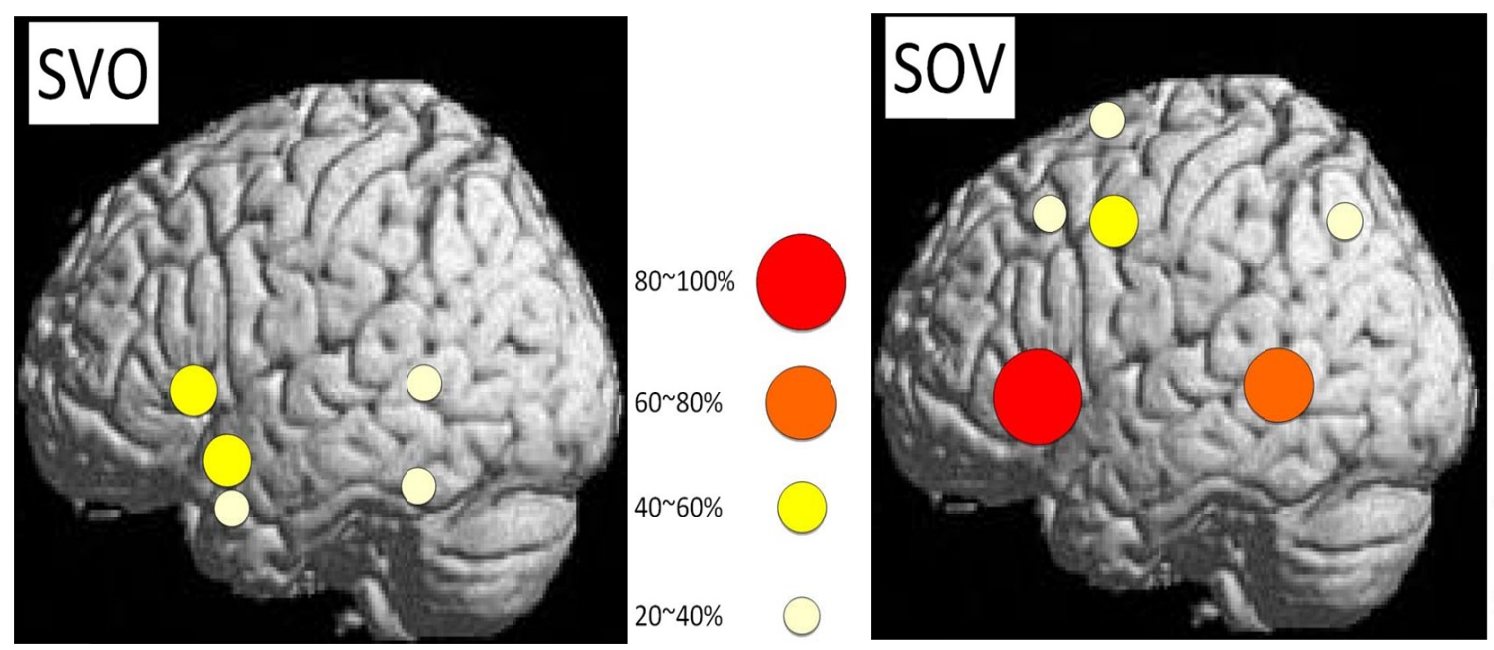

$80 \sim 100 \%$

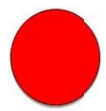

$60 \sim 80 \%$

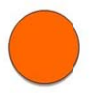

$40 \sim 60 \%$

$20 \sim 40 \%$

Fig. (1). Summary of activation results between SOV and SVO languages.

The upper figure shows the summarized activation results of SOV languages. The right bar shows the ratio, which denotes the number of previous studies reporting the anatomical region as an activated region for 'sentence $v s$. word list' contrast, divided by the total number of previous studies we chose in the current study. In this figure, 100-80\%, 80-60\%, 60-40\%, and 40-20\% are denoted by white, yellow, red, and purple circles, respectively. For visual purposes, only the left hemisphere is shown. The lower figure shows the summarized activation results of SVO languages.

Table 2. Results of Activation Results between SOV and SVO Languages

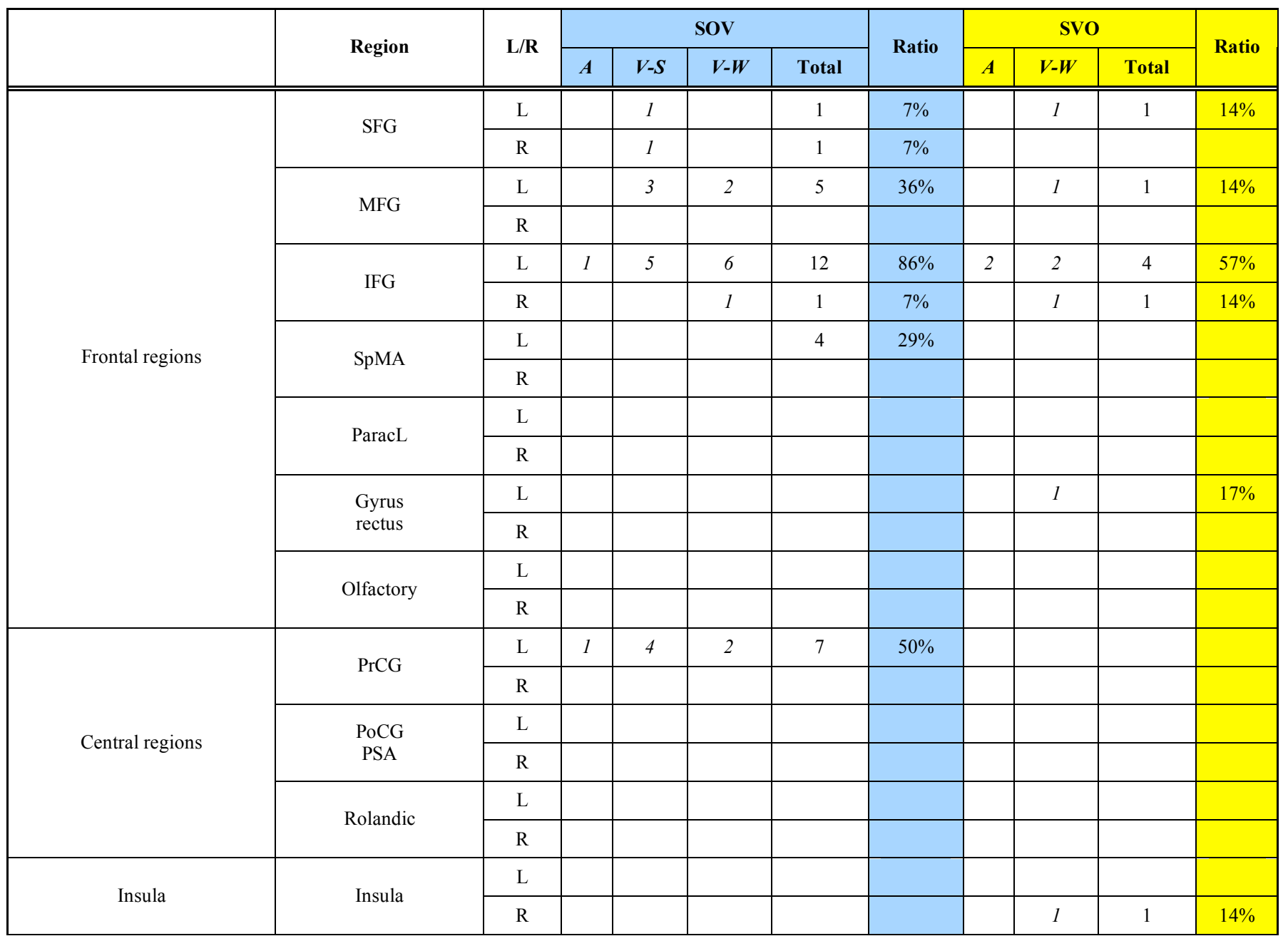


(Table 2) Contd....

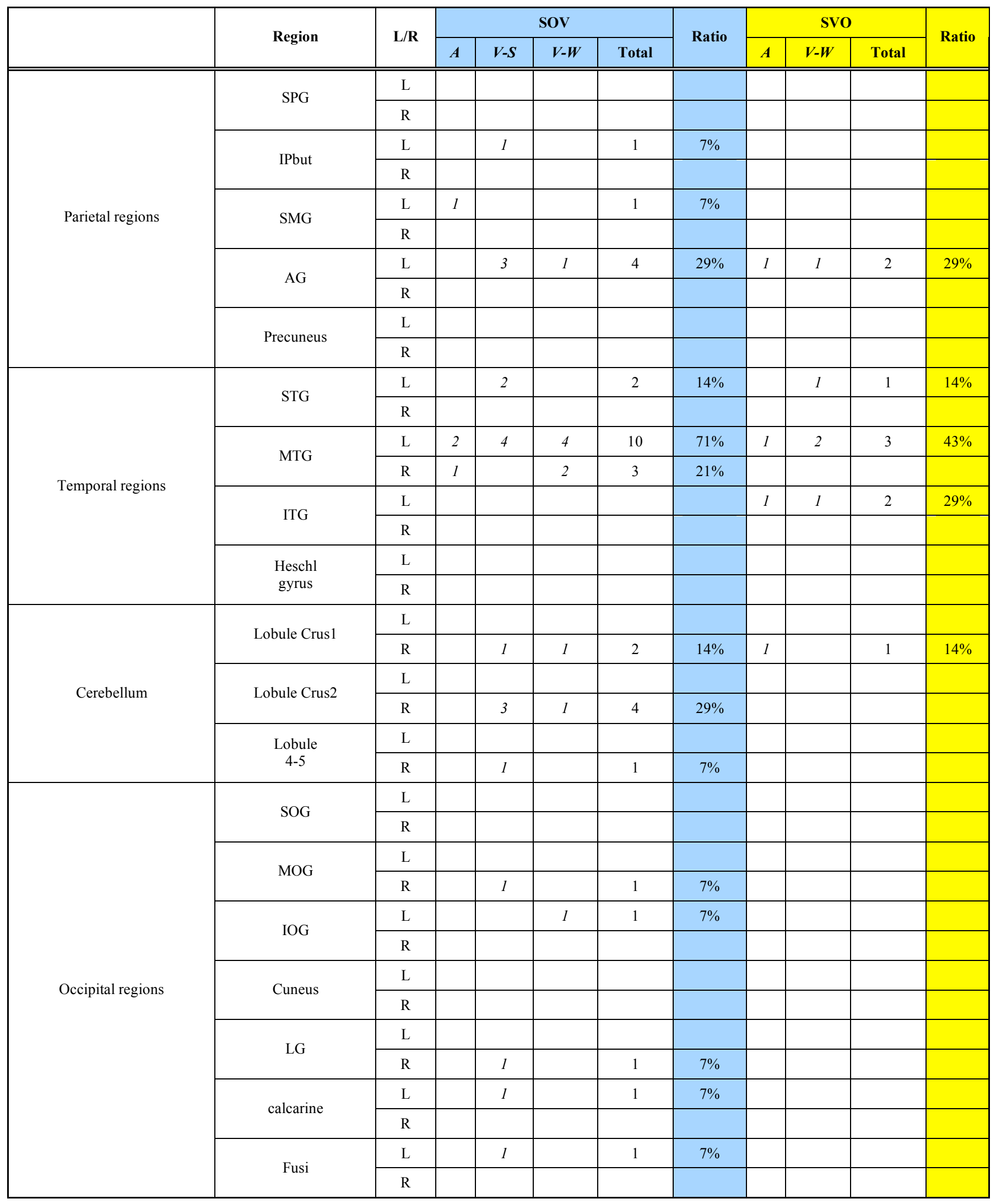


(Table 2) Contd....

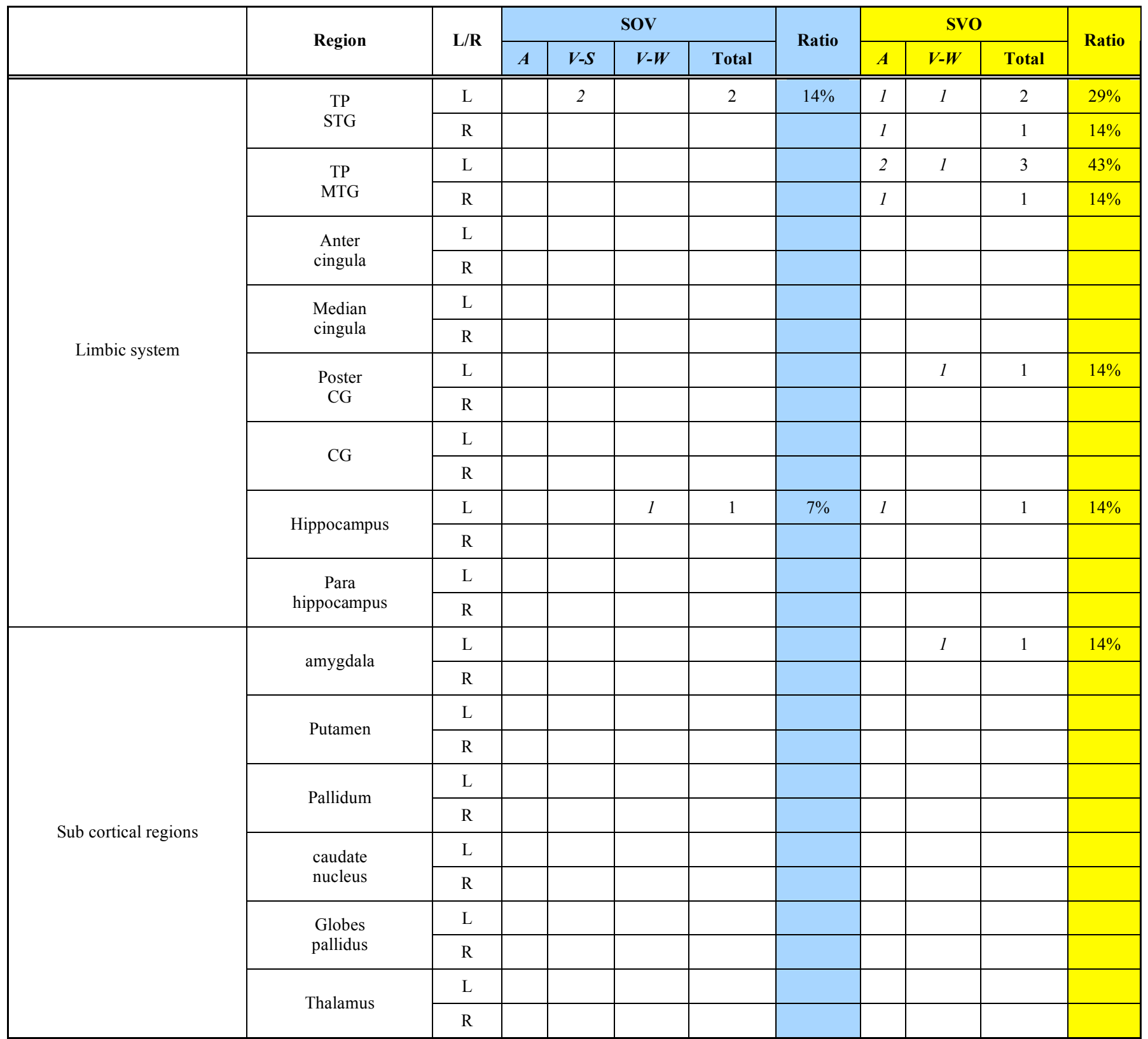

Ratio denotes the number of previous studies reporting the anatomical region as an activated region for the 'sentence $v s$. word list' contrast, divided by the total number of previous studies we chose for the current study. Abbreviations of anatomical brain regions are listed in Table 3 .

Table 3. Abbreviations of Anatomical Brain Regions in Table 2

(Table 3) Contd....

\begin{tabular}{|c|c|}
\hline SFG & Superior Frontal Gyrus \\
\hline MFG & Middle Frontal Gyrus \\
\hline IFG & Inferior Frontal Gyrus \\
\hline SpMA & Supplementary Motor Area \\
\hline ParacL & Paracentral Lobule \\
\hline Gyrus rectus & Gyrus rectus \\
\hline Olfactory & Olfactory Cortex \\
\hline PrCG & Precentral Gyrus \\
\hline PoCG & Postcentral Gyrus \\
\hline Rolandic & Rolandic Operculum \\
\hline
\end{tabular}

\begin{tabular}{|c|c|}
\hline Insula & Insula \\
\hline SPG & Superior Parietal Gyrus \\
\hline IPbut & Inferior Parietal, but Supramarginal and Angular Gyri \\
\hline SMG & Supramarginal Gyrus \\
\hline AG & Angular Gyrus \\
\hline Precuneus & Precuneus \\
\hline STG & Superior Temporal Gyrus \\
\hline MTG & Middle Temporal Gyrus \\
\hline ITG & Inferior Temporal Gyrus \\
\hline Heschl gyrus & Heschl Gyrus \\
\hline
\end{tabular}


(Table 3) Contd....

\begin{tabular}{|c|c|}
\hline SOG & Superior Occipital Gyrus \\
\hline MOG & Middle Occipital Gyrus \\
\hline IOG & Inferior Occipital Gyrus \\
\hline Cuneus & Cuneus \\
\hline LG & Lingual Gyrus \\
\hline Calcarine & Calcarine fissure and surrounding cortex \\
\hline Fusi & Fujiform Gyrus \\
\hline TP STG & Temporal pole: Superior Temporal Gyrus \\
\hline TP MTG & Temporal pole: Middle Temporal Gyrus \\
\hline Anter cingula & Anterior Cingulate and Paracingulate Gyri \\
\hline Median cingula & Median Cingulate and Paracingulate Gyri \\
\hline Poster CG & Posterior Cingulate Gyrus \\
\hline $\mathrm{CG}$ & Cingulate Gyrus \\
\hline Hippo & Hippocampus \\
\hline Para hippo & Parahippocampus \\
\hline Amygdala & Amygdala \\
\hline Putamen & Lenticular nucleus, Putamen \\
\hline Pallidum & Lenticular nucleus, Pallidum \\
\hline Caudate nucleus & Caudate nucleus \\
\hline Thalamus & Thalamus \\
\hline Cerebellum & Cerebellum \\
\hline
\end{tabular}

These anatomical names are based on MARINA software.

\section{DISCUSSION}

In order to investigate whether or not a difference in word order affects functional brain imaging data during sentence comprehension, we used a simple and explorative literature review. Our results suggest that an effect of different canonical word order exists between SVO and SOV languages on brain activation during sentence comprehension. In the following section, we discuss why and how different canonical word order affects brain activation during sentence comprehension between SVO and SOV languages. Our discussion will focus primarily on the left inferior and middle frontal gyri, precentral gyrus, supplemental motor area, inferior and middle temporal gyri, temporal pole, hippocampus, and cerebellum, since these regions show a clear distinction between the SVO and SOV languages (i.e., above a $20 \%$ difference). Also, these results were all leftlateralized, indicating that the results are compatible with the general assumption that language functions are dominated by the left hemisphere. In the following sections, we discuss the reason why the above brain regions are activated differently in SVO and SOV languages.

\subsection{The Inferior Frontal Gyrus}

This result may suggest that the left inferior frontal gyrus plays a role in sentence comprehension. Our results showed that the left inferior frontal gyrus is responsible for different aspects of sentence comprehension in SVO and SOV languages.
Here, we formed two possible interpretations to explain the difference in activation between SVO and SOV languages in the left inferior frontal gyrus. The first is case particle processing. Case particle processing is done in the left inferior frontal gyrus [22]. Actually, most SOV languages have case particles, while SVO languages generally do not have them. The second interpretation is that the load of tentative short-term memory for memorizing arguments may be greater for SOV languages than SVO languages due to a difference in the position of the head, or verb, in a sentence.

Our result suggests that the second interpretation is more plausible than the first. Were the first explanation correct, SVO languages would not activate the left inferior frontal gyrus as SVO languages do not require case particle processing. However, the left inferior frontal gyrus was activated for SVO languages. In contrast, if the second explanation is correct, left inferior frontal activation might be found even in SVO languages since some short-term memory load is necessary in sentence comprehension, even in SVO languages.

\subsection{The Middle Temporal Gyrus}

One possible way of accounting for the different brain activation patterns between the SVO and SOV languages is a different semantic working memory load, since the left middle temporal gyrus has been reported to be related to semantic, or conceptual, working memory [25]. As described above, SOV languages have to maintain the information of the arguments until the input of the verb. This maintenance load may reflect the frequent reports of the existence of activation in the middle temporal gyrus. However, such semantic/conceptual working memory activates not only the middle temporal gyrus, but also the inferior frontal gyrus [23]. Hence, the frequent reports of left inferior frontal activation for SOV languages may also reflect the maintenance load of semantic working memory.

\subsection{The Inferior Temporal Gyrus and Temporal Pole}

This region clearly showed a different activation pattern from the left inferior frontal gyrus. While the left inferior frontal gyrus more frequently activates for SOV languages than it does for SVO languages, the left inferior temporal gyrus is active for SVO languages less frequently than it is for SOV languages. At this time, the role of the left inferior temporal gyrus on sentence comprehension remains unclear; however, one possibility is that the different activation pattern between SVO and SOV languages is caused by a different time course of brain activation for sentence comprehension. Ikuta et al., (2006) [15] showed that left inferior temporal gyrus activation is found when sentence information is integrated. Generally, in SVO languages, since the verb (head) can be used at an early stage of sentence comprehension (i.e., after the subject), the integration process of a sentence may begin at this time. In SOV languages, since the verb (head) can be used at the final stage of sentence comprehension (i.e., final position in a sentence), the integration process of a sentence may not begin until the end of a sentence. Hence, in SOV languages, only when the onset of brain activation is set for the end of a sentence, left inferior temporal gyrus activation may be found, as is demonstrated in Ikuta et al., (2006) [15]. 
Several neuroimaging studies reported that the anterior temporal regions are active during sentence comprehension [24-29]. Particularly, Rogalsky and Hickok (2009) [10] suggested that the anterior temporal region is involved in the semantic integration process from the information of each argument to a sentence's meaning. However, as described above, between SVO and SOV languages, the timing of such semantic integration differs. This difference in timing may affect the different brain activation patterns during sentence comprehension between the two types of languages.

\subsection{The Middle Frontal Gyrus, Precentral Gyrus, Supp- lementary Motor Area, and Cerebellum}

These regions showed similar activation patterns, since these regions were more active for SOV languages than for SVO languages. Hence, these three regions have a similar role in sentence comprehension.

The left dorsal prefrontal cortex, which is near or includes the middle frontal gyrus and precentral gyrus, is known to be related to the executive function of the working memory process. Activation of these regions may be caused by the different working memory processing load between SVO and SOV languages. As described in the Introduction, since in SVO languages the head can determine the entire structure of a sentence, the information given by the head can be used at an early stage in sentence comprehension. Contrastively, since the head is placed at the end of a sentence in SOV languages, the information given by the head cannot be used until the end of the sentence. This word order difference may cause a greater working memory load for the maintenance of arguments in a sentence for SOV languages than for SVO languages. Consistently, our results showed a pattern of greater brain activation related to working memory load (i.e., the left dorsal prefrontal cortex) for SOV languages than for SVO languages. These results are in line with several sentence comprehension models $[30,31]$ which claim that the maintenance of arguments in a sentence for SOV languages may cause a greater working memory load than for SVO languages.

At this time, the role the cerebellum plays in sentence comprehension remains unclear, but several studies claimed that the cerebellum was involved in language comprehension [32]. One account suggested its involvement in the language processing load. This account is in line with our results, as cerebellum activation is similar to the activation of regions related to working memory load (i.e., dorsal prefrontal cortex).

\section{CONCLUSION}

In the current paper, to investigate whether or not the difference in word order affects functional brain imaging data during sentence comprehension, we conducted an explorative literature review. Our results found that differences in canonical word order between SVO and SOV languages affect brain activation during sentence comprehension, mainly in the left inferior and middle temporal gyri, supplementary motor area, precentral gyrus, inferior and middle temporal gyri, temporal pole, hippocampus, and cerebellum. These results suggest that a difference in canonical word order causes a different sentence processing pattern, and also that this difference causes a different load in the working memory process. We discussed why the observed results were found based on our literature review results, but the claim is still speculative. In future studies, it will be necessary to directly examine whether or not cross-linguistic difference affects brain activation for sentence comprehension in different languages and, if so, how.

\section{CONFLICT OF INTEREST}

None declared.

\section{ACKNOWLEDGEMENT}

None declared.

\section{REFERENCES}

[1] Lewis P. Ethonologue: Languages of the world. $16^{\text {th }}$ ed. Dallas: SIL International 2009.

[2] Greenberg JH. Universals of Language - Revised $2^{\text {nd }}$ ed. Cambridge: The MIT Press; 1966.

[3] Hawkins JA. A performance theory of order and constituency. Cambridge: Cambridge University Press 1994; pp. 231-65.

[4] Hawkins JA. Efficiency and complexity in Grammars. USA: Oxford University Press 2004

[5] Kamide Y, Mitchell DC. Incremental pre-head attachment in Japanese parsing. Lang Cognitive Process 1999; 14(5): 631-62.

[6] Miyamoto ET. Case markers as clause boundary inducers in Japanese. J Psycholinguist Res 2002; 31(4): 307-47.

[7] Jeong H, Sugiura M, Sassa Y, et al. Effect of syntactic similarity on cortical activation during second language processing: a comparison of English and Japanese among native Korean trilinguals. Hum Brain Mapp 2007; 28(3): 194-204.

[8] Newman AJ, Supalla T, Hauser P, Newport EL, Bavelier D. Dissociating neural subsystems for grammar by contrasting word order and inflection. Proc Natl Acad Sci USA 2010; 107(16): 753944.

[9] Yokoyama S, Okamoto H, Miyamoto T, et al. Cortical activation in the processing of passive sentences in L1 and L2: an fMRI study. Neuroimage 2006; 30(2): 570-9.

[10] Rogalsky C, Hickok G. Selective attention to semantic and syntactic features modulates sentence processing networks in anterior temporal cortex. Cereb Cortex 2009; 19(4): 786-96.

[11] Humphries C, Binder JR, Medler DA, Liebenthal E. Syntactic and semantic modulation of neural activity during auditory sentence comprehension. J Cogn Neurosci 2006; 18(4): 665-79.

[12] Maguire EA, Frith CD. The brain network associated with acquiring semantic knowledge. Neuroimage 2004; 22(1): 171-8.

[13] Vandenberghe R, Nobre AC, Price CJ. The response of left temporal cortex to sentences. J Cogn Neurosci 2002; 14(4): 550-60.

[14] Bottini G, Corcoran R, Sterzi R, et al. The role of the right hemisphere in the interpretation of figurative aspects of language. A positron emission tomography activation study. Brain 1994; 117 (Pt 6): 1241-53.

[15] Ikuta N, Sugiura M, Sassa Y, et al. Brain activation during the course of sentence comprehension. Brain Lang 2006; 97(2): 15461.

[16] Homae F, Hashimoto R, Nakajima K, Miyashita Y, Sakai KL. From perception to sentence comprehension: the convergence of auditory and visual information of language in the left inferior frontal cortex. Neuroimage 2002; 16(4): 883-900.

[17] Yokoyama S, Yoshimoto K, Miyamoto T, Kawashima R. Neurophysiological evidence of linguistic empathy processing in the human brain: a functional magnetic resonance imaging study. $\mathrm{J}$ Neurolinguistics 2009; 22(6): 605-15.

[18] Kim J, Koizumi M, Ikuta N, et al. Scrambling effects on the processing of Japanese sentences: An fMRI study. J Neurolinguist 2009; 22(2): 151-66.

[19] Homae F, Yahata N, Sakai KL. Selective enhancement of functional connectivity in the left prefrontal cortex during sentence processing. Neuroimage 2003; 20(1): 578-86. 
[20] Hashimoto R, Sakai KL. Specialization in the left prefrontal cortex for sentence comprehension. Neuron 2002; 35(3): 589-97.

[21] Walter B, Blecker C, Kirsch P, et al. MARINA: an easy to use tool for the creation of Masks for Region of Interest Analyses [abstract]. Presented at the 9th International Conference on Functional Mapping of the Human Brain, June 19-22, 2003, New York, NY. Available on CD-Rom in NeuroImage, Vol. 19, No. 2.

[22] Inui T, Ogawa K, Ohba M. Role of left inferior frontal gyrus in the processing of particles in Japanese. Neuroreport 2007; 18(5): 4314.

[23] Fiebach CJ, Friederici AD, Smith EE, Swinney D. Lateral inferotemporal cortex maintains conceptual-semantic representations in verbal working memory. J Cogn Neurosci 2007; 19(12): 2035-49.

[24] Stowe LA, Paans AMJ, Wijers AA, Zwarts F, Mulder G, Vaalburg W. Sentence Comprehension and word repetition: a positron emission tomography investigation. Psychophysiology 1999; 36: 786-801.

[25] Friederici AD, Meyer M, Von Cramon DY. Auditory language comprehension: an event related fMRI study on the processing of syntactic and lexical information. Brain Lang 2000; 74(2): 289300 .
[26] Friederici AD, Von Cramon DY. Syntax in the brain: linguistic versus neuroanatomical specificity. Behav Brain Sci 2000; 23(1): 32-3.

[27] Humphries C, Willard K, Buchsbaum B, Hickok G. Role of anterior temporal cortex in auditory sentence comprehension: an fMRI study. Neuroreport 2001; 12(8): 1749-52.

[28] Humphries C, Love T, Swinney D, Hickok G. Response of anterior temporal cortex to syntactic and prosodic manipulations during sentence processing. Hum Brain Mapp 2005; 26(2): 128-38.

[29] Meyer M, Alter K, Friederici A. Functional MR imaging exposes differential brain responses to syntax and prosody during auditory sentence comprehension. J Neurolinguistics 2003; 16: 277-300.

[30] Bornkessel I, Schlesewsky M. The extended argument dependency model: a neurocognitive approach to sentence comprehension across languages. Psychological Rev 2006; 113(4): 787-821.

[31] Yokoyama S, Yoshimoto K, Kawashima R. Partially incremental triple-pathway model: real time interpretation of arguments in a verb-final Japanese language simplex sentence. Psychology of Language, Nova Science Publisher, to appear.

[32] Pillai JJ, Allison JD, Sethuraman S, et al. Functional MR imaging study of language-related differences in bilingual cerebellar activation. AJNR Am J Neuroradiol 2004; 25: 523-32.

(C) Hashimoto et al.; Licensee Bentham Open .

This is an open access article licensed under the terms of the Creative Commons Attribution Non-Commercial License (http://creativecommons.org/ licenses/by-nc/3.0/), which permits unrestricted, non-commercial use, distribution and reproduction in any medium, provided the work is properly cited. 